\title{
Towards an Epigenetics-Inspired Control System for Power Dispatch Problem
}

\author{
Daniel J. Couvertier, Erik Goodman and Kalyanmoy Deb \\ BEACON Center for the Study of Evolution in Action \\ Michigan State University \\ East Lansing, MI 48824 \\ \{couverti,goodman,kdeb\}@egr.msu.edu
}

\begin{abstract}
Artificial neural networks and genetic and evolutionary optimization algorithms are abstractions of observed natural processes and are found to have a widespread applicability in solving difficult problems. Epigenetics is a biological mechanism in which heritable changes are passed from one generation to another without genetic alterations. These changes allow organisms to adapt to abrupt changes in the environment. We propose and present an epigenetics-inspired learning paradigm illustrated in a power dispatch problem in which generators may fail abruptly. The proposed system is trained on optimized data, and should be able to handle a wider variety of operating conditions. The proposed idea is generic and novel but requires further application to more complex real-world control system problems to fully ascertain its value.
\end{abstract}

\section{CCS CONCEPTS}

- Computer systems organization $\rightarrow$ Neural networks; Reliability - Networks $\rightarrow$ Network design principles; • Computing methodologies $\rightarrow$ Planning under uncertainty;

\section{KEYWORDS}

Epigenetics, Artificial Neural Networks (ANNs), Power Dispatch Problem, Uncertainty

\section{INTRODUCTION}

A great source of inspiration for advances in the artificial world of computing is nature and its biological processes. The observation of evolutionary mechanisms and their emulation in algorithms have given rise to Genetic and Evolutionary Computation which has helped solve global optimization problems. One of these algorithms is the elitist Non-dominated Sorting Genetic Algorithm

Permission to make digital or hard copies of part or all of this work for personal or classroom use is granted without fee provided that copies are not made or distributed for profit or commercial advantage and that copies bear this notice and the full citation on the first page. Copyrights for thirdparty components of this work must be honored. For all other uses, contact the owner/author(s).

GECCO '17 Companion, July 15-19, 2017, Berlin, Germany

ACM 978-1-4503-4939-0/17/07.

http://dx.doi.org/10.1145/3067695.3076104
(NSGA-II) [5], which has become a powerful tool in solving multi-objective optimization problems. Of particular interest to this work, NSGA-II has been utilized to solve power dispatch problems $[1,4]$, where the goal is to operate an electric power system so as to minimize fuel costs while concurrently satisfying other constraints such as environmental considerations.

Apart from evolution, neural networks observed in brains, have inspired the development of Artificial Neural Networks (ANNs), which are also an effective device for developing control systems, including power dispatch problems. Solutions to this problem are commonly found under stable and failure-free conditions where abrupt shutdowns of generators are not taken into consideration. While this is acceptable in most theoretical situations, real-world applications tend to be subject to uncertainty in their dynamic environments.

To manage these particular problems, we look back to biology, where organisms are able to respond well to similarly varying environments thanks to epigenetic factors. These mechanisms allow changes in organisms caused by modifications to the expression of the genetic code rather than by modifying the code itself [2]. In essence, they are a short-term fix for short-term problems that should not affect long-term adaptation. Applications where epigenetics are applied to ANNs are noticeably scarce in the literature. This seems like a missed opportunity given that epigenetics function as a layer of operation above a base system, which would seem to fit perfectly with the layered layout of ANNs.

While evolution allows for adaptation across long periods of time, epigenetics allows for adaptation to specific environmental cues and can return to normalcy once they have been removed. In this paper, we propose to add an epigenetic mechanism as a layer on top of a fully functioning ANN used to solve a power dispatch problem with four generators subject to abrupt failures. We do so by training a modified ANN on a subset of failure conditions, along with normal operation conditions, and epigenetically merging it with an ANN that was trained under normal operation conditions.

\section{PROPOSED EPIGENETIC ANN}

To obtain a solution for the power dispatch problem, we used a setup similar to that employed by Ah King et al. [1] with some modifications. We reduced the system to four thermal generators and focus on a single objective of emphasizing cost and emission 


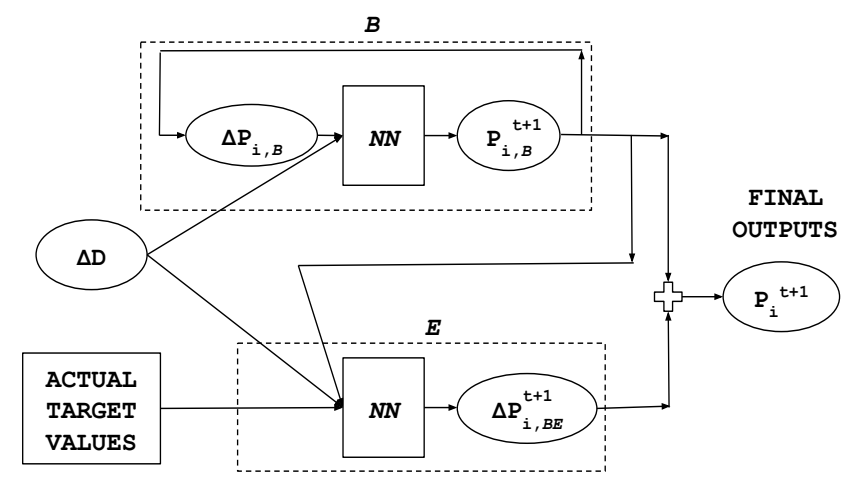

Figure 1: Layout of our Epi-ANN for $i$ generators. $B$ presumes that normal operation conditions are permanent and runs autonomously. $E$ modifies the outputs of $B$ to match those of the overall system which is running under various failure conditions.

equally (later studies will involve multi-objective considerations). NSGA-II was utilized to find feasible solutions over a simulated 90-day period where the load demand varied over time, Solutions were generated for cases where all generators are operating normally and where each generator was individually shut down.

The ANNs are designed and implemented using the Matlab Neural Network Toolbox [6] and trained using the backwardpropagation-of-errors method. Performance is measured using mean squared error (MSE). Various instances of ANNs are trained under the case of all generators operating normally, and others under cases of failure in order to have optimized systems to compare the epigenetic setup against.

To implement our Epigenetic ANN (Epi-ANN), we first train a standard ANN under normal operation conditions, where all generators are always online and operating correctly, and use it as a base $B$ upon which we add our epigenetic layer $E$. Since $B$ is assumed to be operating under normal conditions, its only external input is the change in load demand from $t-1$ to $t(\Delta D)$. Its outputs, the power generated by each generator $i$ at the end of the timestep $t\left(P_{i}^{t+1}\right)$, are fed back as inputs for the next timestep, allowing $B$ to run autonomously. $E$ is trained under adverse conditions, such as when generators abruptly shut down, which renders the set $P_{i}^{t+1}$ produced by $B$ incorrect. $E$ takes as inputs $\Delta D, B^{\prime}$ s outputs, and the set of target values as provided by NSGA-II. E's output is the set of differences that are required in order to have the outputs of $B$ match the targets provided by NSGA-II. Figure 1 shows a schematic of our approach.

\section{RESULTS AND DISCUSSION}

We have completed the implementation of the Epi-ANN and have found promising results. The MSE for the Epi-ANN indicates that the approach works on the power dispatch problem and, under failure conditions, it is capable of outcompeting standard ANNs. We also explored how the composition of the training data affects the performance of the ANNs. For standard ANNs, including more failure conditions in the training data allows them to handle abnormal operation conditions better at the expense of decreased performance under normal conditions. For the Epi-ANN, including normal operation conditions in the training data improves performance at no apparent cost, allowing it to activate when anomalies are present, and to 'silence' itself when operation is normal. Natural epigenetics were able to develop under a delicate balance of abrupt short-term changes within slow long-term changes, and our system should be granted the same balance within the conditions it is being exposed to. More detail about this epigenetics-inspired control system can be found in our technical report [3].

In this work, we proposed and presented a working prototype for an Epigenetic ANN as a controller for a power dispatch problem. Preliminary results indicate that the approach is able to respond well to time-varying normal and sudden changes, all while returning quickly to regular operation when the system gets back to normalcy. We have argued that biological epigenetic mechanisms work in a similar manner and our implementation stays as an epigenetic mimicry for addressing abrupt changes to artificial control systems.

As immediate follow-ups to this work, we wish to perform extensive runs to establish a statistically significant difference between our method and other possible ANNs and control strategies. Furthermore, we wish to explore and define the training parameters that are necessary to get the most out of our approach, including, but not limited to, the composition and the size of the training data for each case given the dissonant nature of the systems that we need to ultimately compare on equal terms. Nevertheless, this initial study has been motivating to us to evaluate the proposed Epi-ANN and its variants on more complex control system problems.

\section{ACKNOWLEDGMENTS}

This material is based in part upon work supported by the National Science Foundation under Cooperative Agreement No. DBI-0939454. Any opinions, findings, and conclusions or recommendations expressed in this material are those of the author(s) and do not necessarily reflect the views of the National Science Foundation.

\section{REFERENCES}

[1] Robert T. F. Ah King, Harry C. S. Rughooputh, and Kalyanmoy Deb. 2005. Evolutionary Multi-objective Environmental/Economic Dispatch: Stochastic Versus Deterministic Approaches. Springer Berlin Heidelberg, Berlin, Heidelberg, 677-691. DOI: http://dx.doi.org/10.1007/978-3-540-31880-4_47

[2] Warren W. Burggren and David Crews. 2014. Epigenetics in Comparative Biology: Why We Should Pay Attention. Integrative and Comparative Biology 54, 1 (2014), 7. DOI:http://dx.doi.org/10.1093/icb/icu013

[3] D.J. Couvertier, E. Goodman, and K. Deb. 2017. An Epigenetics-Inspired Control System Based on Artificial Neural Networks: Results on a Power Dispatch Problem. COIN Report Number 2017014. Technical Report. Michigan State University, East Lansing, MI.

[4] K. Deb. 2008. Scope of stationary multi-objective evolutionary optimization: A case study on hydro-thermal power dispatch problem. Journal of Global Optimization 41, 4 (2008), 479-515.

[5] K. Deb, A Pratap, S. Agarwal, and T. Meyarivan. 2002. A fast and elitist multiobjective genetic algorithm: NSGA-II. IEEE Transactions on Evolutionary Computation 6, 2 (Apr 2002), 182-197. DOI:http://dx.doi.org/10.1109/4235.996 017

[6] Howard Demuth and Mark Beale. 2009. Matlab neural network toolbox users guide version 6. The MathWorks Inc. (2009). 